\title{
FORMACIÓN TEOLÓGICA Y MINISTERIO PASTORAL
}

DOI: https://doi.org/10.52039/seminarios.v51i175.733

\begin{abstract}
Autor: Tullio Citrini. Rector de la Pontificia Universidad Gregoriana de Roma y Rector del Colegio Lombardo.
\end{abstract}

Tradujo. A. Morata.

Revisó: Juan Manuel Cabiedas
El ministerio plasma la orientación de fondo de la vida sacerdotal. Una formación dirigida a capacitar 'para' el ministerio, y a potenciar el interés apostólico. Un estudio de la teología que impulse a salir de sí mismo y asimilar lenguajes y formas diferentes, adoptando el nivel de las personas a evangelizar.

La cuestión formulada en el título de esta reflexión constituye el segundo cuadro de un díptico, el primero del cual ha sido la conferencia del profesor Sandro Panizzolo sobre Formación teológica y persona del pastor. El sentido de esta doble visión viene dado en la misma articulación de la Presbyterorum ordinis (el ministerio y la vida de los presbíteros). En esta semana de estudio el discurso sobre teología y vida de los presbíteros ha precedido al de la teología y el ministerio.

El orden inverso de los dos temas en Presbyterorum ordinis fue establecido deliberadamente: tratar el ministerio antes que la vida del presbítero apunta a una elección fundamental del concilio a propósito de esta cuestión: el ministerio ha de plasmar la orientación de fondo de la vida del sacerdote, ha de darle una significatividad específica. El ministerio es razón de vida para el sacerdote; la vida del sacerdote está dedicada a aquel. 
Naturalmente la tarea formativa debe tener en cuenta esta orientación, debe formar sacerdotes dedicados al ministerio, cuya vida esté al servicio de la Iglesia y de su misión. Pero el itinerario formativo debe considerar igualmente no sólo este aspecto objetivo sino también el punto de partida, es decir, los propios jóvenes que se preparan para el sacerdocio. La capacidad de tales jóvenes para una vida cristiana madura ha de ser comprendida en ese período de preparación, incluso puede decirse que es anterior a esa capacitación para el ejercicio del ministerio.

De aquí brotan una serie de interrogantes sobre los que trataremos de reflexionar en lo que sigue.

\section{1. ¿Qué ocurre cuando el estudio de la teología se desarrolla desde la apertura a los otros?}

\subsection{Primera cuestión: ¿tiempos diferentes?}

De cuanto hemos dicho hasta ahora parecería deducirse una respuesta positiva. Sin embargo, pongamos sobre el tapete dos fenómenos generales, aunque no se presenten de la misma manera en las distintas iglesias: los seminarios menores van desapareciendo o han desaparecido totalmente, y la formación catequética de los adolescentes y jóvenes en las parroquias o en los variados grupos cristianos resulta a menudo superficial e insuficiente; esto hace que quien llama a las puertas del seminario mayor (bien sea en la etapa de filosofía o teología, como quiera que se estructuren en concreto) tenga generalmente una fuerte necesidad de consolidación de su fe, no digamos en lo referente a la propia inteligencia teológica. Dicha necesidad ha de urgir un mejor conocimiento de la revelación y la teología en su dimensión de servicio a los demás; cuando una tal percepción de la tarea teológica no existe en el período de formación, puede dar lugar al hecho de que el celo por la evangelización del prójimo se sustente en una amplia ignorancia de la doctrina de la fe, felizmente inconsciente.

En menor medida se da el fenómeno opuesto. A la puerta del seminario mayor o directamente a la de los obispos se presentan personas que han hecho ya un itinerario consistente de formación teológi- 
ca, habiendo incluso alcanzado el grado de bachiller en teología u otros títulos. En este caso la dificultad reside en el hecho de que ni obispos ni seminarios sabe muy bien qué hacer con estas personas durante los cuatro años que el Código prescribe como obligatorios; de todos modos la cuestión de fondo es otra bien distinta.

Volviendo al fenómeno que más se da hoy: En general un primer momento del tiempo de seminario exige atención a la formación de personas creyentes, siendo sucesiva la evidencia de una formación específica del futuro pastor. Esto mismo es válido en lo que se refiere a la formación intelectual. Puede resultar especialmente útil un tiempo propedéutico, sobre todo en aquellos lugares en los que estén netamente diferenciados los estudios filosóficos y teológicos. Alternativamente, puede resultar igualmente útil un bienio mixto de filosofía y teología, porque una inmersión en el camino puramente fílosófico puede resultar una ducha de agua fría para aquellos que evidencian una viva tensión espiritual. Con la vista puesta en esta posible introducción Karl Rahner ideó su Curso fundamental sobre la fe; con ocasión de la quaestio disputata sobre La reforma de los estudios teológicos había mostrado ya su propósito en este sentido así como un proyecto relativo al mismo.

Tanto por razones de tipo pedagógico como de tipo didáctico, no es posible hacer una distinción excesivamente rígida de los objetivos. Los temas de la fe y la teología son evidentemente idénticos, y no tendría sentido pasar por ellos dos veces. Esta visión general y la vuelta subsiguiente sobre lo que se debe enseñar de las diversas materias tiene sentido en las escuelas de grado inferior al universitario, porque el crecimiento global de niños y adolescentes reclama que se hagan calas progresivas en los temas y se les presenten además síntesis de los mismos; pero en el ámbito de los estudios universitarios ne bis in idem. Además quien ingresa en el seminario, aun cuando tenga todavía problemas personales en lo referente a la asunción y clarificación de la fe, declara y se le supone un interés apostólico explícito, que debe seguir cultivándose en un delicado equilibrio con la gratuidad absoluta de la pasión por la verdad y el bien.

Naturalmente no tendría sentido responder a la pregunta sobre los ritmos si la respuesta no fuese positiva, al menos de alguna manera, a la cuestión relativa a los contenidos y al aprendizaje. 


\subsection{Segunda cuestión: ¿Una teología diferente?}

Como pura formalidad, no. La inteligencia de la fe tiene una lógica propia que no puede adaptarse sin más a diversas finalidades. En los años en torno a la Segunda Guerra Mundial hubo un intento de elaborar, en contraste con la rigidez de la neoescolástica, una segunda teología llamada "kerigmática", más inspirada en la Biblia, en la liturgia y en la experiencia espiritual. El episodio es muy conocido en la historia de la teología del siglo pasado: aquella propuesta fracasó, pero dejó como herencia una serie de exigencias que el mismo Vaticano II reconoce como fundamentales para toda teología, dentro de un amplio margen de pluralismo teológico.

Se percibe igualmente que la presencia de un determinado interlocutor hace variar necesariamente la escena. Si esto sucede, generándose una segunda teología, o diversas teologías secundarias y paralelas, es porque no existe la fe al margen de su propia comunicación, ni ésta sin interlocutores. Estos no son un añadido irrelevante. Es cierto que la verdad de Dios transciende los lenguajes del hombre, pero la revelación no se da prescindiendo u obviando tales lenguajes.

\subsection{Tercera cuestión: ¿un ejercicio formativo diverso?}

Estudiar teología con la perspectiva explícita de un servicio a la fe de otros constituye un ejercicio de virtud nada despreciable, ya sea intelectual o relacional: ambas son virtudes típicas de la personalidad global del pastor, ámbitos que la caridad pastoral mantiene unidos. Me permito citar algunas páginas de un texto bien conocido por mí porque he tomado parte en su redacción. Las cito porque me parecen suficientemente claras y me eximen del esfuerzo de repetir de otro modo, quizás menos cuidadoso, las mismas cosas. Se trata del proyecto formativo del seminario de Milán, publicado en 1995 en la revista La Scuola Cattolica e impreso en un volumen independiente por la editorial Ancora bajo el título: La formación del presbítero diocesano. Líneas educativas del seminario de Milán. En la sección referente al cuatrienio teológico, titulada La formación del pastor para el ejercicio del ministerio, que está a continuación de la relativa al primer bienio del seminario mayor con el título: La formación del creyente orientada al 
ministerio presbiteral, se llega a hablar en un cierto punto específicamente del estudio de la teología.

Se señala la necesidad del estudio de la teología por parte de aquellos creyentes que se preparan para el presbiterado, de la preciosa sinergia entre el vivir y el saber de la fe; después, más minuciosamente, en el número 83 del texto se dirige:

A aquellos que se dedican al ministerio se les pide el paciente y ponderado estudio del saber teológico, que posee una metodología propia, sus articulaciones y sus dinámicas, requiriendo igualmente muchos esfuerzos, que no se exigen de por sí a todo creyente. La teología, en el camino de preparación al ministerio, persigue el objetivo de la apropiación de los lenguajes, los modelos, los itinerarios argumentativos de la revelación divina y de su transmisión en la fe de la iglesia, a favor y al servicio de esta misma fe. Por tanto, es necesario el cultivo humilde y entusiasta en el inestimable tesoro compuesto por los documentos de la fe revelada, su conocimiento cierto, la habilidad interpretativa, la capacidad comunicativa. Al final del cuatrienio teológico cada uno debería encontrarse en condiciones de constatar que la propia fe se ha abierto a las dinámicas objetivas de la Palabra de Dios, vivida e interpretada dentro de la tradición de la fe eclesial. Esta estructuración puede suponer para cada uno el poder realizar una composición armónica, entre apropiación personal y objetiva de la fe comprendida. Se trata de una adquisición decisiva para la serenidad del propio camino ministerial, donde se «sabe» la verdad de Jesús como fundamento indiscutible de la propia vocación y como realidad intangible del propio servicio a la palabra de la predicación. Esta es condición esencial para un ministerio que no se centra en sí mismo, y atestigua con gratitud ante los otros la verdad del Dios de Jesús. Un ministerio, que sepa integrar armónicamente la tensión entre creer y comprender, y no interprete e imagine esta tensión, tolerada y susceptible de olvido, como el trabajo acotado a la ya pasada etapa del seminario, podrá defenderse de los peligros opuestos, desgraciadamente muy difundidos, de un pragmatismo agobiante o de un árido intelectualismo.

Y más adelante, en el número 85 se subraya:

En positivo, el estudio de la teología requiere un esfuerzo para salir de sí mismo, del propio esquema mental y cultural de origen, una docilidad a esa verdad integral, con la cual sólo se puede servir a 
la comunidad creyente; la asimilación de lenguajes y modos de pensar diferentes, una adecuada capacidad de discernimiento de la propia época. El cuidado de la comunidad local exige todo esto, de modo que el ministerio sea servicio a la objetividad de un cristianismo que soporta mal ser reducido o adulterado dentro de perspectivas parciales. El estudio de la teología, el esfuerzo inevitable que conlleva, la lentitud de sus ritmos, su proceder metódico, el consiguiente trabajo intelectual, deben ser entendidos como un momento de la pasión por el Evangelio y sus destinatarios. La atención a la vida de estos últimos, la finura en el trato, la capacidad de escucha, la entrega a su historia particular, la consideración del punto de vista de los otros, forman parte del cultivo en la capacidad de escucha y de diálogo con la tradición creyente, asimilando en el múltiple testimonio de la fe la actitud tenaz del creyente para dar crédito a la verdad de Dios que Jesús nos comunica. Así la teología educa a través de su mismo aprendizaje, no sólo como introducción consciente a los contenidos de la fe, sino porque da razón del acto de creer: de aquí deriva el servicio al Evangelio, que puede ser transmitido a los otros, porque a su vez nosotros lo hemos recibido como un tesoro precioso. Los currículos de la escuela y del estudio son como las vasijas de arcilla que portan este tesoro; éstos son un «inútil servicio» al futuro ministro del Evangelio, para que de ninguna manera se sienta dueño, sino siervo fiel de la verdad de Dios, de la que hace participar incesantemente el Espíritu de Jesús.

La cuidada estructura de este texto, mientras abre horizontes interesantes de espiritualidad pastoral, revela a la vez la relación entre formación personal en la fe a través de la teología y formación para la fe a través de la misma teología con vistas al ministerio. El texto reproducido permite observar que las virtudes requeridas en uno y otro camino son en gran medida idénticas, de suerte que en concreto se trata de un único camino en la formación del creyente pastor. Puede añadirse que si no fuese así quedaría desmentida la tesis fundamental de Presbyterorum Ordinis referente a la primacía del ministerio en la definición de la identidad del presbítero.

En el centro de este análisis se encuentra la identidad de la actitud espiritual y cultural de escucha de la revelación y del prójimo. Quien sabe o aprende a escuchar la Palabra de Dios, sabe y aprende a 
escuchar a las personas; y viceversa, la disponibilidad, la paciencia, la inteligencia, la flexibilidad necesaria para escuchar al prójimo son premisas indispensables para la fe, para la inteligencia teológica de la misma, así como para su apropiada comunicación.

No se nos escapa que en la experiencia del estudio hay momentos de dificultad e incluso de objeción; hay pasajes en el estudio teológico que son poco gratificantes, del mismo modo que a menudo es poco gratificante la dedicación al prójimo, y en cualquier caso no lo es automáticamente. Hay una dimensión ascética en el estudio, que puede ser vivida con más aspereza por aquel que está poco inclinado a un trabajo intelectual, pero que llega también a las inteligencias más vivaces y especulativas. El «esfuerzo por salir de sí mismo, del propio esquema mental y cultural», para ponerse al servicio de legítimos modos de pensar de otros, de horizontes culturales que deben ser evangelizados y bendecidos, pero que no es fácil ni instintivo compartir por el pastor o por el futuro pastor, es tarea que promete a la larga su recompensa, como todo esfuerzo que dilata el pensamiento, pero ciertamente no viene por sí, sino sólo de una fe que se fortalece recorriendo en la caridad pacientes y metódicos itinerarios de entendimiento.

En esta perspectiva es muy instructivo darse cuenta hasta qué punto la compleja lección del pasado es preciosa para prepararse a afrontar el complejo desafío del presente y de un próximo futuro. De poco serviría recorrer una y otra vez la historia de la teología planteando problemas teológicos ya superados, a no ser que se pretenda aprender cuales son los verdaderos caminos trazados por Dios en medio de nuestra humanidad compleja, ambigua y pecadora; o bien establecer indicadores que apunten los caminos del futuro.

\section{La "totalidad" necesaria e imposible}

En vistas a una formación no sectorial, unilateral, potencialmente "herética" de la inteligencia de la fe, las directrices de la Iglesia vienen insistiendo en la "visión completa y unitaria" de la formación teológica, sobre todo de los pastores. Valga en este sentido el primer párrafo de Pastores dabo vobis, n. 54: 
La formación teológica es una tarea sumamente compleja y comprometida. Ella debe llevar al candidato al sacerdocio a poseer una visión completa y unitaria de las verdades reveladas por Dios en Jesucristo y de la experiencia de la fe de la Iglesia, de ahí la doble exigencia de conocer «todas» las verdades cristinas y conocerlas de manera orgánica, sin hacer selecciones arbitrarias. Esto exige ayudar al alumno a elaborar una síntesis que sea fruto de las aportaciones de las diversas disciplinas teológicas, cuyo carácter específico alcanza auténtico valor sólo en la profunda coordinación de todas ellas.

Todo esto se encuentra con una dificultad evidente, que obliga a preguntarse cuál es el significado real de esta exigencia propuesta por la Iglesia. La dificultad proviene de hecho de que la visión unitaria y total de la teología desde el punto de vista material es, en cuanto tal, imposible, y ni siquiera constituye un objetivo sensato. La revelación sobrepasa siempre nuestra inteligencia y capacidad de síntesis. El problema se ha presentado así en la Iglesia desde la antigüedad: el canon de las Escrituras, los símbolos de la fe, los catecismos oficiales responden a la preocupación por una visión de unidad equilibrada y tendencialmente completa de la fe cristiana. ¿Pero qué significa esto hoy, qué sobreentiende la exigencia a que apunta la comunidad eclesial?

Será necesario buscar una respuesta adecuada ya que sobre esta búsqueda de equilibrio y unidad se cargan presiones unilaterales y desafortunadas. En efecto, muchos creen, ingenuamente pero con firme$\mathrm{za}$, que lo mejor para promover este legítimo interés del cual son guardianes, es proponer el añadido de nuevos cursos en el currículo de los seminarios. Pero el resultado no puede ser sino una sobrecarga, además de un evidente desequilibrio ya que tal iniciativa está sustentada por una lógica ajena a un auténtico conocimiento de causa.

\subsection{Nuestros interrogantes y los de la gente}

La distinción y el lazo de unión establecido entre intereses personales y ministeriales en el estudio de la teología lleva a la conclusión de que la unidad y complexión de una formación teológica encuentra su medida tanto en nuestros interrogantes como en los del resto de personas. Puesto que vivimos en una cultura compleja y plural, dichos interrogantes son diversos, de modo que el horizonte de la deseada visión unitaria se alarga bastante. 
La atención a las inquietudes de las personas es importante. Allí donde éstas no se escuchan falta el estímulo para la reflexión, faltan instrumentos para elaborarla. Quien no sabe escuchar la pregunta, no sabe a su vez proponerla, y viceversa. Si se elimina todo esto, la teología desaparece. Se constata esto mismo allí donde la comunidad cristiana permanece inerte y desinteresada. Además, sólo el hecho de que el interés práctico por un determinado tema de la revelación se difumine u oscurezca, comporta que la teología lo afronte más como una cuestión abstracta y de escuela, relegándolo, poco a poco, al olvido. La teología se hace siempre para alguien.

\subsection{Las cuestiones que la gente no se formula o se propone mal}

Ahora bien, no puede olvidarse que la revelación se inicia en Dios y la teología no puede por tanto seguir solamente los intereses de la persona. Una buena formación teológica debe ayudar a los pastores a estimular aquellas cuestiones que la revelación suscita y que aproximan a la misma, así como orientar el interés de la persona a través de las provocaciones del evangelio. A menudo las cuestiones mal formuladas o guiadas por intereses no limpios, son obstáculo para la escucha de la Palabra de Dios, el encuentro con El y su salvación. Alejarse de las « discusiones vanas que no conducen a nada» $(2$ Tim 2,14$)$ es compromiso del hombre de Dios. Desenmascarar preguntas del tipo: ies verdad que Dios ha dicho: no debéis comer de ningún árbol del jardín?, o ¿es lícito a un hombre repudiar la propia mujer por cualquier motivo?, exige una sabiduría pastoral que se forja a través de un atento aprendizaje teológico. Además, es acertado subrayar que objetivo de la teología no es tanto suministrar respuestas sobre Dios y la revelación cuanto ayudar a plantear correctamente las preguntas relativas a ello. Unidad y totalidad no significa ciertamente responder a todas las preguntas, ni siquiera a las mal planteadas; ante éstas últimas se impone como reacción la denuncia.

\subsection{Las tareas típicas del ministerio del presbítero}

Una sabiduría acorde con la revelación es útil a todo cristiano, y una formación catequética madurada a través de una verdadera y pro- 
pia teología es preciosa para todos, aun cuando en diversa medida y manera. Desenmascarar las preguntas falsas es normalmente tarea del pastor, pero a menudo pertenece más a un carisma profético que pastoral. No faltan todavía tareas típicas del ministerio presbiteral, cuya consideración puede también estimular inflexiones que afectan al estudio de la teología.

Pienso en la salvaguardia de la memoria de Jesús, que ha sido confiada a aquel que ha sido escogido como ministro del evangelio y para conservar a la comunidad en la eucaristía (gratitud y sacramento) y la misma Eucaristía (sacramento y gratitud) en la comunidad. A los apóstoles se les ha encomendado el hacer discípulos a todos los pueblos, bautizándoles en el nombre del Padre, y del Hijo y del Espíritu Santo, enseñándoles a observar todo lo que Jesús ha mandado. En este paso de la doctrina a la praxis, de la memoria a la praxis, es bueno que la teología aprendida por quien desarrolla el ministerio presbiteral se muestre dispuesta y atenta. El servicio de los pastores a la palabra de Dios no puede consistir sólo en proclamar una doctrina que posteriormente, para cada cristiano o grupo de cristianos, en el desarrollo de la responsabilidad autónoma de las diversas vocaciones y de los diversos carismas del Espíritu, carezca de la necesaria incidencia en su historia concreta y su vida personal. Ni por el contrario puede pretender dictar minuciosamente los comportamientos cristianos, especialmente en ámbito secular. Sin embargo, sí supone ciertamente una función de guía en la relectura y reescritura continua del sentido concreto de los mandatos de Dios, que actuando en combinación con los dones que el Espíritu distribuye para el bien común según su inexcusable libertad, recuerda a la Iglesia, y a través de ella a toda la humanidad, a qué clase de libertad estamos llamados, qué felicidad se nos ha prometido, qué amor nos mueve, y qué cuidadoso discernimiento debemos ejercer, a qué exigente seguimiento hemos sido llamados, cuán suave y ligero es el yugo de Cristo llevado con Él.

Pienso ahora en el ministerio de la comunión para el cual son habilitados y llamados los ministros sagrados por el sacramento del orden. Para que no desemboque en una imperceptible unidad interior, la comunión eclesial y la reconciliación de la humanidad de la que ella es signo e instrumento, deben adquirir la forma de una comunicación de lenguajes que dé continuidad, en la complejidad hoy marcada por la 
globalización de todo fenómeno, de todo proceso y cultura, al signo del primer Pentecostés. Por supuesto sin repetir las formas espectaculares de la primera efusión del Espíritu santo, un acontecimiento de esta clase se presenta constantemente con los rasgos del milagro y la utopía. Fruto de una gracia inalcanzable con las solas fuerzas humanas, tiende a una plenitud que sólo la vuelta del Señor Jesús pueđ̉e satisfacer. Pero ha de ser cultivada, al punto de poder ser señalada al menos como esperanza, como posibilidad real a través de realizaciones quizás pequeñas, a través de vivencias comunitarias reconocibles como verdaderas y realmente habitables. Me parece necesario que la teología que se enseñe a los candidatos al ministerio presbiteral se haga cargo de la exigencia de que éstos sean iniciados en esta responsabilidad por la comunicación de y en la fe.

\section{El diálogo con la gente más instruida}

En fin, un sacerdote debe ser capaz de dialogar también con gente muy instruida, a veces más que él mismo. Personas especializadas en áreas que ellos no cultivan, sensibles a cuestiones a las que ellos no pueden dedicarse. El tiempo en el cual el clero constituía el culmen de la formación cultural de la población a ellos confiada, a la par que el médico, el farmacéutico, el abogado, el maestro de escuela, es ya tiempo pasado. Y ello dado que se va haciendo realidad en todo el mundo la extensión de la educación básica, que puede prolongarse en especializaciones muy concretas; otra razón menos afortunada es que la afición a una formación intelectual de calidad ha sido abandonado por una parte del clero. Limitémonos a considerar aquí dos situaciones que pueden presentarse con cierta frecuencia.

\subsection{Cuando los fieles suscitan dificultades "cultas"}

El sacerdote puede encontrarse con fieles que planteen, con total legitimidad, cuestiones "cultas", o muy técnicas. Puede deberse al hecho de que cada vez con más frecuencia nuevas áreas interpelan el juicio moral: es hoy típico el caso de la bioética. O porque la difusión más o menos precisa de ideologías y religiones exóticas presentan 
cuestiones inquietantes para la gente. O porque la fe tiende a ser cada vez menos algo propio de gente "ruda" (que es otro de los pobres del evangelio). Los motivos pueden ser muchos más.

En general, los verdaderos especialistas, sabedores de la complejidad y el carácter técnico del propio saber, que interpelan a la fe con cuestiones surgidas de su específico campo de competencia, saben que no pueden dialogar sobre ello con el primer sacerdote que se encuentren. Pero mientras la vida cotidiana no tiene reparo alguno en plantear una gran cantidad de preguntas surgidas de áreas diversas, la complejidad de las disciplinas teológicas (exegéticas, historiográficas, sistemáticas, prácticas) es difícilmente imaginable para aquellos que no las frecuentan en alguna medida. Sucede así que muchas personas, sabedoras del grado de especialización de la propia competencia, no imaginan el equivalente en el saber de la fe, de modo que piensan que cualquier hombre de Dios debe estar en disposición de responder a cualquiera de las cuestiones.

En este contexto el sacerdote tiene indudablemente el derecho de no saber responder a todo. Lo tiene como cualquiera, porque es una regla general de juego de las competencias en la cultura contemporánea. Es interesante notar que este derecho es mejor asumido por quien más sabe, porque se da cuenta más fácilmente de los límites del propio saber y del saber humano en general. En la famosa "Quaestio disputata". La reforma de los estudios teológicos (trad. italiana, Morcelliana, Brescia 1970), Karl Rahner escribía así:

Quisiera enunciar la tesis, que quizás suene un poco maligna, aunque retengo exacta si se tiene en cuenta la cuestión por la cual nosotros, en la situación actual, que he intentado delinear brevemente, somos todos rudos en un cierto sentido necesaria e inevitablemente, y podemos serlo pacífica y animosamente e incluso mostrarnos también ante el mundo como tales (p.79).

Él mismo por otra parte, en el mismo ensayo, había hecho precisamente a propósito de nuestra pregunta una precisión de esta guisa:

A un sacerdote no debe serle transmitido necesariamente todo aquello que deberá saber más tarde en el ámbito de su programa de formación, a través de las materias y las enseñanzas obligatorias. La hipótesis contraria lleva a un excesivo gravamen del plan de estudios (p. 38). 
Y también esta otra:

En conjunto, sin embargo, la formación básica debe tener una orientación práctica y existencial y por tanto un principio constructivo y selectivo: el cristiano y el sacerdote de hoy en su existencia personal y el pastor normal, que puede habérselas igualmente con la persona culta del momento, y no el joven erudito especialista es el objetivo de la formación de base (p. 40).

Habérselas significa, si lo entiendo bien, saber orientarse y saber orientar a los fieles en las cuestiones que se refieren a la fe, saber recurrir a quien está más especializado según la naturaleza de las preguntas que se afrontan cuando no se sepa dar una respuesta personalmente. Si en general se enseña a los seminaristas, que cuando el día de mañana se puedan encontrar perdidos por alguna dificultad de naturaleza canónica o administrativa, a qué sección de la curia dirigirse, ¿por qué no, en sentido análogo, debería formar parte de la formación normal del presbítero saber cómo situarse frente a dificultades de naturaleza teológica? O quizás, puesto que saben a quién dirigirse, ¿por qué no formar para que se perciba como normal esta conjunción entre pastores genéricos y especialistas (clérigos o laicos), sin renunciar al gusto y al deber de saberse mover autónomamente donde sea posible, según la utilidad de los fieles?

Puede resultarnos simpático leer una interesante página de Santo Tomás, aunque la situación actual sea diversa a la contemporánea a éste teólogo. Entonces se planteaba un problema particular para los sacerdotes que no tenían la responsabilidad de la cura de almas; hoy es difícilmente pensable una ordenación en semejante perspectiva. El santo doctor se planteaba la cuestión de si a aquellos que reciben las órdenes se debe exigir el conocimiento de toda la Sagrada Escritura (Summa Theologiae, Suppl, q. 36, a. 2 = in IV Libr. Sententiarum, dist. 24, a.3, q.1-2) Y respondía:

El sacerdote tiene una doble actividad: una principal, sobre el cuerpo de Cristo verdadero, y otra secundaria, sobre el cuerpo místico de Cristo. La segunda actividad depende de la primera, pero no viceversa. Por esa razón son promovidos al sacerdocio algunos a quienes se les confía solamente la primera actividad, como los religiosos a quienes no se les confía la cura de almas. De la boca de estos no se busca la ley, sino sólo que celebren los sacramentos. Para estos 
es suficiente que sepan lo necesario para poder observar correctamente lo que atañe a la celebración del los sacramentos. Otros en cambio son promovidos a la otra actividad, que es sobre el cuerpo místico de Cristo. De su boca el pueblo espera la ley. Por eso en ellos debe estar presente el conocimiento de la ley. No en el sentido de que conozcan todas las cuestiones difíciles de la ley, porque para esto debe recurrirse a los superiores; sino que sepan de la ley lo que el pueblo debe creer y observar. Pero a los sacerdotes superiores, esto es a los Obispos, compete saber también las cosas que en la ley pueden generar dificultad; y tanto más cuanto más alto es el puesto en que han sido colocados.

\subsection{Cuando los fieles saben más teología que los pastores}

En su tiempo santo Tomás consideraba normal que el obispo fuera especialmente competente en todas las cuestiones referentes a la comprensión de la Escritura. Es más, al margen del reconocimiento de la autoridad de la cathedra pastoralis, probablemente el mismo Tomás no se hacía muchas ilusiones sobre la ciencia de los obispos, salvo que los considerase en comparación con la media de los presbíteros en la tarea de la cura de almas. Tomás personalmente pertenecía a una orden particularmente docta, y trabajaba en ambientes universitarios.

Hoy tres cuartos de milenio después, el cuadro es más intrincado. Siguiendo la serie de situaciones que nos planteábamos al inicio de este apartado, se puede considerar como el último caso el de los fieles que saben más teología que los pastores. Es tal vez un caso no infrecuente, sobre todo donde se ha difundido más la cultura teológica de los laicos; y no es un caso falto de dificultad en la medida que los presbíteros se esfuerzan por superar la situación embarazosa que se les puede plantear. El problema reproduce a menor escala el dilatado debate sobre las relaciones entre la teología y el magisterio; aun a menor escala constituye todo un problema de pastoral y de espiritualidad más que de teología fundamental.

El camino a recorrer es evidentemente el de valorar con gratitud los dones de todos, orientándolos al bien de la Iglesia. Para obtener este resultado el sacerdote no deberá por supuesto afrontar todas las eventuales especializaciones de los fieles que se le confían. Es bueno 
sin embargo que sepa moverse con suficiente inteligencia y agilidad, para no sucumbir al temor o a los celos, para saber intuir el eventual riesgo que se esconde en especializaciones plagadas de "lobos rapaces", saber custodiar la responsabilidad inalienable de guía de la comunidad que reconoce los dones del Espíritu y promueve su natural destino al bien común.

Curso ARCER, 19 noviembre 2003 